\title{
QUESTIONÁRIO DE QUALIDADE DE VIDA NA EPILEPSIA
}

\section{Resultados preliminares}

\author{
Elisabete Abib Pedroso de Souza'
}

\begin{abstract}
RESUMO - Crises epilépticas acontecem por breves períodos de tempo, mas os sentimentos de ansiedade e desamparo, adaptação às restrições impostas aos estilos de vida e uma variedade de outros problemas afetam mais a qualidade de vida (QV) dos portadores de epilepsia. Este estudo propõe um protocolo que contempla os objetivos de uma avaliação de QV na saúde e seja aplicado a uma população que frequenta um hospital universitário. Consta de 65 itens e avalia o funcionamento psicossocial, limitações físicas, aspecto cognitivo, percepção de controle, auto-conceito, bem como percepção de saúde e QV. Os resultados de validação e confiabilidade são discutidos.
\end{abstract}

PALAVRAS-CHAVE: qualidade de vida, protocolos, epilepsia, adultos.

Protocol of quality of life in epilepsy: first results

ABSTRACT - Seizures happen for brief periods of time, but the feelings of anxiety and helplessness, adaptation to the restrictions impose by the life-style and variety of other problems affect more the quality of life in epilepsy. This study proposes a protocol that regards the objectives of a quality of life assessment in health and is applied to a population that attends a university hospital. It contains 65 items and assesses the psychosocial performance, physical limitation, cognitive aspects, perception of control, self-concept, as well as perception of health and quality of life. The results of reliability and validity are discussed.

KEY WORDS: quality of life, epilepsy, adults, protocol.

\section{Conceito de qualidade de vida}

Questões relacionadas ao bem-estar subjetivo começaram a ganhar importância na área de saúde quando a expectativa de vida fez aumentar a incidência de doenças crônicas, mostrando a necessidade de se avaliar o impacto dessas condições na rotina dos pacientes. Uma perspectiva médica tende a focalizar intensamente os resultados clínicos decorrentes da avaliação de sintomas e sinais da doença. Hoje, ouvir o que o paciente tem a dizer sobre o que sente faz parte de uma avaliação integral do estado da doença, dos efeitos adversos do uso de medicamentos e da análise dos efeitos de procedimentos terapêuticos. Qualidade de vida (QV) é um conceito subjetivo, baseado na perspectiva do sujeito. O paciente é a pessoa que melhor pode definir sua própria QV e relatar sobre suas expectativas, satisfação e bem estar. Calman ${ }^{1}$ discute o conceito de QV como a diferença entre as experiências reais e as expectativas. Assim, o impacto na vida de dois indivíduos com idênticos tipos de epilepsia e resposta ao tratamento (i.e., frequência, severidade e efeitos adversos) pode diferir, dependendo de como cada pessoa percebe as restrições ou perdas de oportunidades no dia a dia. Cramer ${ }^{2}$ estabelece que o balanço entre o status percebido e o desejado é a essência da equação de QV. A epilepsia, então, afeta cada pessoa diferentemente porque envolve crenças, expectativas e percepções que são categorias intra-psíquicas individuais relacionadas com a história de vida de cada um. Assim, auto-estima, independência, educação, problemas para dirigir, emprego, medo das crises, etc, são problemas da vivência da condição epiléptica. A Organização Mundial de Saúde (OMS) definiu saúde como um estado de completo bem estar físico, mental e social ${ }^{3}$. A classificação da OMS de comprometimento, déficit e prejuízo também esclarece questões de QV quando associa comprometimento a um parecer clínico enquanto o paciente focaliza mais seus prejuízos ${ }^{4}$. QV nas doenças

Departamento de Neurologia da Faculdade de Ciências Médicas (FCM) da Universidade Estadual de Campinas (UNICAMP) Campinas SP, Brasil: ${ }^{1}$ Psicóloga, Professor Assistente Doutor.

Recebido 22 Novembro 2000, recebido na forma final 26 Março 2001. Aceito 11 Abril 2001.

Dra. Elisabete Abib Pedroso de Souza - Departamento de Neurologia, FCM/UNICAMP - Caixa Postal 6111 - $13083-970$ Campinas SP Brasil.E-mail: assouza@bestway.com.br 
crônicas é reconhecida como um conceito multidimensional pois fala de problemas da vivência da condição epiléptica, que vão além dos sintomas da doença e fazem parte da experiência do sujeito.

\section{Modelos de investigação de QV}

Foram propostos numerosos modelos e desenvolvida uma variedade de medidas para avaliar QV na doença crônica ${ }^{5-6}$. Há inventários mais gerais como The Sickness Impact Profile (SIP) ${ }^{7}$, The General Health Questionnaire $(\mathrm{GHQ})^{8}$, The Nottingham Health Profile (NHP) ${ }^{9}$, e Rand 36-Item Health Survey ${ }^{9}$, bem como inventários mais específicos relativos às doenças investigadas.

Na epilepsia, embora muitas variáveis psicossociais tenham sido investigadas, somente nas últimas décadas foram agrupadas no conceito de QV. Esse estudo não pretende fazer uma revisão dos protocolos existentes, somente fazer referências àqueles que tiveram significância nessa área. Uma revisão crítica foi feita recentemente por Sander e O'Donoghue ${ }^{10}$ e Leidy et al ${ }^{11}$. O primeiro estudo sistemático na área foi desenvolvido por Dodrill et al. ${ }^{12}$ que, apesar de ser considerado um passo na quantificação de auto-relatos, acabou não preenchendo muitos critérios de avaliação de $\mathrm{QV}$ relacionados à saúde abordados pelas pesquisas sociais nas últimas décadas ${ }^{13}$.

Com o objetivo de avaliar a severidade das crises, são citadas três escalas. A primeira, criada em 1978 pelo Department of Veterans Affairs (VA) Cooperative Study of Antiepileptic Drugs ${ }^{14}$; e as Escalas de Gravidade de Crises de Chalfont ${ }^{15}$ e de Liverpool ${ }^{16}$. Os estudos de Chaplin et al. ${ }^{17}$, Collings ${ }^{18}$ e Jacoby ${ }^{19}$ desenvolvem pesquisas com populações de portadores de epilepsia com objetivo de identificar problemas relacionados a QV e de estabelecer relações entre as variáveis da doença e as sociais.

O desenvolvimento do Epilepsy Surgery Inventory (ESI-55) por Vickrey et al. ${ }^{20}$, como medida da efetividade da lobectomia anterotemporal, representou uma tentativa para produzir uma avaliação de QV breve e adequada. Foi importante por incorporar uma medida genérica que permite comparações com outras doenças e medidas específicas da epilepsia. O Quality of life in Epilepsy Inventory (QOLIE-89) ${ }^{21}$ surgiu como uma extensão do ESI-55, procurando ser mais abrangente. Estes mesmos autores, com o objetivo de serem mais parcimoniosos, desenvolveram o Quality of life in Epilepsy Inventory com 10 e 31 itens $^{22-23}$.

O'Donoghue et al. ${ }^{24}$, preocupados com aspectos metodológicos relacionados às medidas de $\mathrm{QV}$, propõem o Subjective Handicap of Epilepsy - SHE, usan- do como referencial teórico o conceito de "handicap", foi definida como a desvantagem pessoal, imposta pela doença, de viver o papel social que a maioria considera normal ou desejável. $\mathrm{Na}$ intenção de desenvolver um questionário de fácil aplicação, que pudesse ser respondido por pacientes que frequentam hospitais universitários, foi elaborado o Questionário de Qualidade de Vida-65 (QQV-65).

\section{A metodologia das medidas de resultados}

São necessários cuidados metodológicos criteriosos quando se pretende avaliar resultados complexos, tais como incapacidade e bem estar pessoal ${ }^{10}$. A escolha do instrumento deve se adequar ao objetivo proposto e as condições às quais serão expostas. O primeiro passo é ter um construto teórico que conceitue a medida e, posteriormente, a preocupação que este instrumento seja confiável e validado para a população sob investigação. Existem duas formas para testar a confiança da escala: a consistência interna e reprodutibilidade (confiança testereteste), sendo que a primeira é estimada através do coeficiente alfa de Cronbach que reflete a covariação dos itens com a escala, e a segunda através da correlação intraclasses.

A validação de construção deve diferenciar os grupos ou variáveis sob investigação, e a validação de conteúdo mostra se o instrumento reflete aspectos importantes de QV na população investigada e se as escalas são representativas das áreas físicas, psicológicas e sociais avaliadas.

\section{MÉTODOS}

Sujeitos: Esta avaliação tem por meta atingir uma população de 300 sujeitos que tenham sido diagnosticados como portadores de epilepsia. Esse estudo avalia os primeiros resultados com uma população de 60 sujeitos que frequentaram o ambulatório de epilepsia do HC-UNICAMP no ano de 1999. Tinham idades de $18-70$ anos $(M=37,05$; $\mathrm{DP}=11,25)$, com média de duração da doença igual a 25,68 anos $(4-48$ anos; $\mathrm{DP}=11,28)$ e média de idade de início das crises igual a 11,17 anos $(0-48$ anos; $D P=10,86)$. Destes sujeitos $25(43,9 \%)$ apresentam crises parciais secundariamente generalizadas (CPG), $20(35,1 \%)$ crises tônico-clonica generalizadas (CTCG) e $12(21,0 \%)$ crises parciais. Foram excluídos pacientes com retardo mental evidente, doenças psiquiátricas e aqueles sob uso de medicação (que não drogas anti-epilépticas) que pudessem afetar o sistema nervoso central.

Instrumento: Questionário de Qualidade de Vida 65 (QQV-65). Este questionário foi elaborado a partir da análise dos trabalhos de Vickrey et al. ${ }^{20}$, Devinsky et al. ${ }^{21} \mathrm{e}$ $\mathrm{O}^{\prime}$ Donoghue ${ }^{24}$. Consta de duas partes. A primeira compreende identificação pessoal, e relaciona dados demo- 
Tabela 1. Estatística descritiva das pontuações total e por aspecto.

\begin{tabular}{lccccc}
\hline Aspecto & \multicolumn{5}{c}{ Estatística Descritiva } \\
\hline MÉDIA & DP & MAX & MEDIANA & MIN \\
\hline Físico & 61.40 & 21.08 & 100.00 & 66.67 & 0 \\
Social & 53.82 & 16.94 & 85.96 & 52.63 & 15.79 \\
Afeto & 56.19 & 18.63 & 97.22 & 58.33 & 22.22 \\
Percepção de Controle & 60.65 & 22.83 & 94.44 & 61.11 & 16.67 \\
Auto conceito & 63.02 & 21.17 & 100.00 & 66.67 & 16.67 \\
Cognitivo & 63.36 & 29.70 & 100.00 & 66.67 & 0 \\
Total & 61.50 & 14.33 & 90.24 & 63.94 & 30.12 \\
\hline
\end{tabular}

gráficos (idade, data de nascimento, escolaridade, tipo e tempo de emprego, estado civil) e informações relacionadas à doença (tipo de crise, início, duração, frequência, medicação e percepção de controle de crises). Ressalto aqui a diferença entre frequência de crises (número real de crises, expresso em dias, meses e anos) e percepção de controle de crises (avaliação subjetiva do paciente a respeito de como percebe o controle das mesmas).

A segunda parte refere-se ao instrumento propriamente dito que investiga, através de 65 questões, a QV do paciente epiléptico. Avalia percepção de saúde e QV (10 itens), limitação física (5 itens), aspectos sociais (6 itens), satisfação (6 itens), percepção de controle (6 itens), aspectos afetivo-emocionais (12 itens), auto-conceito (8 itens), aspectos cognitivos (4 itens), trabalho (5 itens), lazer (2 itens) e dirigir veículos automotores (1 item).

Este questionário procurou considerar as limitações linguísticas já identificadas em estudos desenvolvidos em nosso meio ${ }^{25}$, relativos a dificuldades de compreensão das questões relacionadas sensivelmente a déficits de escolaridade e confusão na discriminação de diferentes intensidades de respostas. O QQV-65 procurou resolver estas dificuldades através de uma validação semântica, bem como igualou as intensidades de respostas. O QQV-65 é um instrumento utilizado na rotina de atendimento a pacientes portadores de epilepsia no setor de psicologia aplicada à neurologia do HC-UNICAMP.

\section{RESULTADOS}

Estatística descritiva - Todas as escalas foram transformadas linearmente em escalas de 0-100 pontos, com altos valores representando um funcionamento melhor ou bem-estar. Médias (M), desvios padrão (DP), percentagens com escore máximo e mínimo foram calculados para cada escala (Tabela 1).

Avaliação de validade e confiabilidade - A consistência interna para QQV-65 para o escore total e dimensões foi alta (Tabela 2). O coeficiente alfa de Conbrach foi de 0,897 para todos os itens e variou de 0,73 a 0,87 nas diferentes dimensões. A estabilidade temporal do questionário foi estudada através do coeficiente de correlação intraclasses nos pacientes que não relataram mudanças na sua saúde (Tabela 2). Os coeficientes de correlação variaram de 0,772 até $0,940(p>0,05)$. Nenhuma diferença foi encontrada entre os escores médios em ambas as administrações.

Os itens selecionados usados neste questionário respondem aos critérios de validação de conteúdo, pois são representativos dos aspectos importantes da QV e já foram altamente identificados na literatura e abordados nos instrumentos em uso. Somase ainda a experiência de 20 anos de atendimento clínico psicológico desta população pela pesquisadora. Houve também o cuidado de se testar ou rever as respostas dos pacientes através de questões abertas, procedimento já utilizado anteriormente ${ }^{20}$.

A validação de construção foi feita procurando examinar a eficiência das medidas de QV comparando grupos com epilepsia controlada (mais de 150 dias sem crise) e não controlada segundo as respostas dos pacientes quanto ao controle das crises (percepção de controle de crises). A hipótese sob a investigação foi que o primeiro grupo respondesse como tendo melhor qualidade de vida. Este estudo foi analisado em outra publicação. $O$ teste MannWhitney identificou um $p=0,005$ comprovando a

Tabela 2. Coeficientes de Cronbach e de correlação intraclasse para teste da confiabilidade.

\begin{tabular}{lccc}
\hline Dimensões & Cronbach & $\begin{array}{c}\text { Corr. } \\
\text { Intraclasse }\end{array}$ & P-Valor \\
\hline Físico & 0.734 & 0.844 & 0.0005 \\
Social & 0.807 & 0.940 & 0.0001 \\
Emocional & 0.872 & 0.814 & 0.0017 \\
Afeto & 0.791 & 0.803 & 0.0016 \\
Percepção de Controle & 0.731 & 0.773 & 0.0032 \\
Auto conceito & 0.744 & 0.920 & 0.0001 \\
Cognitivo & 0.810 & 0.772 & 0.0032 \\
Total & 0.897 & 0.907 & 0.0001 \\
\hline
\end{tabular}


hipótese. A comparação de QV com a variável emprego (estar empregado ou não) mostrou através do teste Mann-Whitney, um $p$ significante para as dimensões de afeto $(p=0,011)$ e social $(p=0,007)$ mas não para o escore total. A comparação de qualidade de vida com escolaridade não foi significante. Entretanto, devemos considerar que foi feita uma comparação, na população estudada, do primeiro grau completo com o segundo grau incompleto.

\section{DISCUSSÃO}

Estes dados mostraram o QQV-65 como um instrumento sensível e abrangente para avaliar QV na população de pacientes epilépticos que frequentam o HC/UNICAMP. As características psicométricas foram avaliadas por procedimentos estatísticos que contemplaram aspectos de validação e conteúdo, consistência interna e reprodutibilidade. Este teste foi aplicado verbalmente para que se pudesse ter certeza de que o sujeito compreenderia o que estava sendo questionado e foi completado em 20-30 minutos, provendo informações a respeito de aspectos que representaram a satisfação e percepção de bem estar nas áreas física, psicológica e social.

A preocupação com a questão de diferenciar a percepção do sujeito sobre o controle de crises da frequência real de crises abre a possibilidade de se investigar questões subjetivas do controle do bem estar psicológico já identificados por Wilson et al. ${ }^{26}$ e Gopinath et al. ${ }^{27}$.

A Qualidade de Vida assume atualmente importância, porque, mesmo que as crises sejam infrequentes e breves, o efeito adverso das drogas antiepilépticas e as consequências sociais e psicológicas das crises e da epilepsia são persistentes e devastadoras.

Assim, a importância do relato do paciente na avaliação de QV vem cada vez mais consolidar a relevância do conceito da metodologia direcionada para o entendimento dos efeitos psicológicos e sociais, além dos efeitos físicos da epilepsia.

Agradecimentos - Agradecemos a colaboração dos Profs. Drs. Carlos A.M. Guerreiro e Fernando Cendes do Departamento de Neurologia da FCM/UNICAMP responsáveis pelo ambulatório de epilepsia, bem como de Helymar da Costa Machado, Mestre, pelo tratamento estatístico. Comissão de Estatística da FCM/UNICAMP.

\section{REFERÊNCIAS}

1. Calman KC. Quality of life in cancer patients an hypothesis. J Med Ethics 1984:10:124-127.
2. Cramer JA. A clinimetric approach to assessing quality of life in epilepsy. Epilepsia 1993;34(Suppl.4):S8-S13.

3. World Health Organization. World Health Organization: The first 10 years of the World Health Organization. Geneva, Switzerland:World Health Organization, 1958.

4. Schipper H, Clinch J, Powell V. Definitions and conceptual issues. In Spilker B (ed). Quality of life assessment in clinical trials. New York: Raven Press, 1990;11-24

5. Spilker B. Quality of life assessments in clinical trials. New York: Raven Press, 1990.

6. Shumaker S, Anderson R, Czajkowski S. Psychological tests and scales. In Spilker B (ed). Quality of life assessment in clinicals trials. New York: Raven Press, 1990.

7. Bergner M, Babbit RA, Carter WB, Gilson BS. The sickness impact profile: development and final revision of a health status measures. Med Care 1981;19:787-805.

8. Godberg D. Manual of the general health questionnaire. Windsor, England: NEFR Publishing, 1979

9. Hunt SM, McKenna SP, McEwan T, et al. A quantitative approach to perceived health status: a validation study. J Epidemiol Commun Health 1980;34:281-286.

10. O'Donoghue MF, Sander JWAS. Qualidade de vida e gravidade das crises como medida de resultados em epilepsia. In Costa JC, Palmini A, Yacubian EM, Cavaleiro EA (eds). Fundamentos neurobiológicos das epilepsias. São Paulo: Lemos, 1998;2:1321-1348

11. Leidy NK, Rentz AM, Grace EM. Evaluation health-related quality of life outcomes in clinical trials of antiepileptics drugs therapy. Epilepsia 1998;39:965-977.

12. Dodrill CB, Batzel LW, Queisser HR, Temkin NR. An objective method for the assessment of psychological and social problems among epileptics. Epilepsia 1980;21:123-135.

13. Antonak RF, Livneh H. A review of research on psychosocial adjustment to impairment among persons with epilepsy. J Epilepsy 1992;5:194-205

14. Cramer JA, Smith DB, Matson RH, Delgado-Escueta VA, Collings JF and The VA Epilepsy Cooperative Group. A method of quantification for the evaluation of antiepileptic drug therapy. Neurology 1983;33:S26-37.

15. Duncan JS, Sander JWAS. The Chalfont seizure severity scale. J Neurol Neurosurg Psychiatry 1991;54:873-876.

16. Baker GA, Smith DF, Dewey M, Morrow J, Gramford PM, Chadwick DM. The development of a seizure severity scale as an outcome measure in epilepsy. Epilepsy Res 1991;8:245-251.

17. Chaplin JE, Yepes E, Shorvon S, Floyd M. A quantitative approach to measuring the social effects of epilepsy. Neuroepidemiology 1990;9:151-158.

18. Collings JA. Psychosocial well-being and epilepsy: an empirical study. Epilepsia 1990;31:418-426.

19. Jacoby A. Epilepsy and the quality of everyday life: findings from a study of people with well-controlled epilepsy. Soc Sci Med 1992;34:657-666.

20. Vickrey BG, Hays RD, Graber J, Rausch R, Engel JJr, Brook RH. A healthrelated quality of life instrument for patients evaluated for epilepsy surgery. Med Care 1992;30:299-319.

21. Devinsky O, Vickrey BG, Cramer J e al. Development of the quality of life in epilepsy inventory. Epilepsia 1995;36:1080-1104.

22. Cramer JA, Perrine K, Devinsky O, Meador K. A brief questionnaire to screen for quality of life in epilepsy: The QOLIE-10. Epilepsia 1996;37:577-582.

23. Cramer JA, Perrine K, Devinsky O, Bryant-Constock L, Meador K, Hermann B. Development and cross-cultural translation of a 31-item quality of life in epilepsy inventory (QOLIE-31). Epilepsia 1998; 39:81-88.

24. O'Donoghue MF, Duncan JS, Sander JWAS. The subjective handicap of epilepsy. A new approach to measuring treatment outcome. Brain 1998;121:317-343.

25. Souza EAP, Sadat MA, Matos EP. The application of the WPSI in epileptic patients with a low social class. Epilepsia 1997;38(Suppl.7):34.

26. Wilson SJ, Saling MM, Lawrence J, Bladin - Outcome of temporal lobectomy:expectation and the prediction of perceived success. Epilepsy Res 1999;36:1-14.

27. Gopinath B, Radhakrishnam K, Sarma PS, Jayachandrran K, Alexander A. A questionaire survey about doctor- patient communication, compliance and locus of control among South Indian people with epilepsy. Epilepsy Res 2000;39:73-82. 